\title{
NUMERICAL STUDY OF THE MICROSTRUCTURE OF A DILUTE SUSPENSION TO ASSESS ITS THIXOTROPIC BEHAVIOR BY A TWO-WAY COUPLING SCHEME
}

\author{
TOMOHIRO FUKUI, MISA KAWAGUCHI \& KOJI MORINISHI \\ Department of Mechanical Engineering, Kyoto Institute of Technology, Japan
}

\begin{abstract}
It is important to comprehend the microstructure of a suspension in a pressure-driven flow to control its rheological properties. The microstructure is usually assessed based on spatial arrangement of the particles, i.e., how suspended particles flow in a channel. The spatial patterns in the width direction rather than those in the axial one are more important because near-wall particles may increase the equivalent intrinsic viscosity significantly. The viscous dissipation near the channel wall, which is directly related to the pressure drop, depends on how and where suspended particles flow in a channel. A thixotropic behavior of a suspension is partly due to these microstructure changes; however, it has not been completely clarified yet. Therefore, the aim of this study is to consider microstructure changes of a dilute suspension attributed to the inertial effects of the suspended particles, and its accompanying macroscopic rheology changes by a two-way coupling scheme. Pressure-driven suspension flow simulations were conducted by regularized lattice Boltzmann method. A periodic boundary condition was applied in the axial direction of the channel to reduce computational costs. The suspended particles were assumed to be rigid, neutrally buoyant, and chemically stable. The flow simulations were then performed by up to 100 nondimensional time to consider temporal changes in the microstructure. As a result, the microstructure of a suspension was observed to change in time mainly due to inertial effects of the suspended particles. The thixotropic behavior of a dilute suspension due to inertial effects was successfully reproduced by considering changes in its microstructure.
\end{abstract}

Keywords: rheology, non-Newtonian property, thixotropy, particle migration, dilute suspension, two-way coupling simulation.

\section{INTRODUCTION}

Suspension is one of the promising functional fluids to achieve smart control of rheological properties by changing its microstructure. Microstructure of a suspension is usually defined by the positions of the suspended particles relative to each other [1]. Since suspension microstructure dictates its macroscopic rheology, it is important to consider all factors that influence microstructure. When microstructure of a dilute suspension is totally homogeneous, i.e., suspended particles are homogeneously dispersed, its relative viscosity $\eta_{\text {eff }} / \eta_{0}$ can be estimated by Einstein's viscosity equation [2]:

$$
\frac{\eta_{\mathrm{eff}}}{\eta_{0}}=1+[\eta] \phi
$$

where $\eta_{\text {eff }}$ is the effective viscosity of a suspension, $\eta_{0}$ is the viscosity of a particle-free fluid, $[\eta]$ is the intrinsic viscosity, and $\phi$ is the concentration of a suspension. Note that intrinsic viscosity $[\eta]$ is well known to be 2 for $2 \mathrm{D}$ [3] and 2.5 for $3 \mathrm{D}$ [2]. Influence of spatial arrangement of the particles on the rheology, however, has not been well considered yet, and may be important to control its rheological properties. When suspended particles are not dispersed homogeneously, i.e., particles flow heterogeneously, Einstein's equation cannot be applied to estimate its relative viscosity. Doyeux et al. [4] considered that the viscosity of a suspension increases when the suspended particles approach the channel wall, and vice versa. 
Therefore, it is important to consider the temporal changes in microstructure of a suspension so as to assess the macroscopic rheological properties of a suspension in detail.

So far, we have proposed a two-way coupling numerical scheme to evaluate the particle rotation effects on the rheological properties [5]. However, these studies were conducted on the condition that suspended particles were homogeneously distributed in order to satisfy an assumption in Einstein's equation. For better practical applications, suspended particles should be scattered randomly in a channel. The aim of this study is, therefore, to consider the effects of microstructure, which is formed by spatial arrangement of the particles, on the rheological properties of a suspension. Especially, inertial effects are focused in this study because it is one of the major factors to influence microstructure of hard-sphere suspensions.

\section{METHODS}

\subsection{Computational models}

Pressure-driven suspension flow simulations were carried out in a two-dimensional channel. The channel had a length $L(0<x<\mathrm{L})$ of $1620 \mu \mathrm{m}$ and a width $2 l(-l<y<l)$ of $400 \mu \mathrm{m}$, respectively. Note that periodic boundary conditions in the axial direction were taken into consideration to reduce computational costs. As illustrated in Fig. 1, suspended particles, with a diameter $2 r$ of $20 \mu \mathrm{m}$, were randomly distributed in the channel as an initial condition. The confinement $C$, which is the ratio of particle size $2 r$ to channel width $2 l$, was consequently set $0.05(=20 / 400)$. This parameter is important when particle inertia is considered. The concentration $\phi$ of the suspension was $1.02 \%$, which corresponds to totally 21 particles in a computational domain, for a dilute assumption. The particles were also assumed to be rigid, non-colloidal, and chemically stable. Moreover, their density was set equivalent to that of the suspending fluid to satisfy neutral buoyancy.

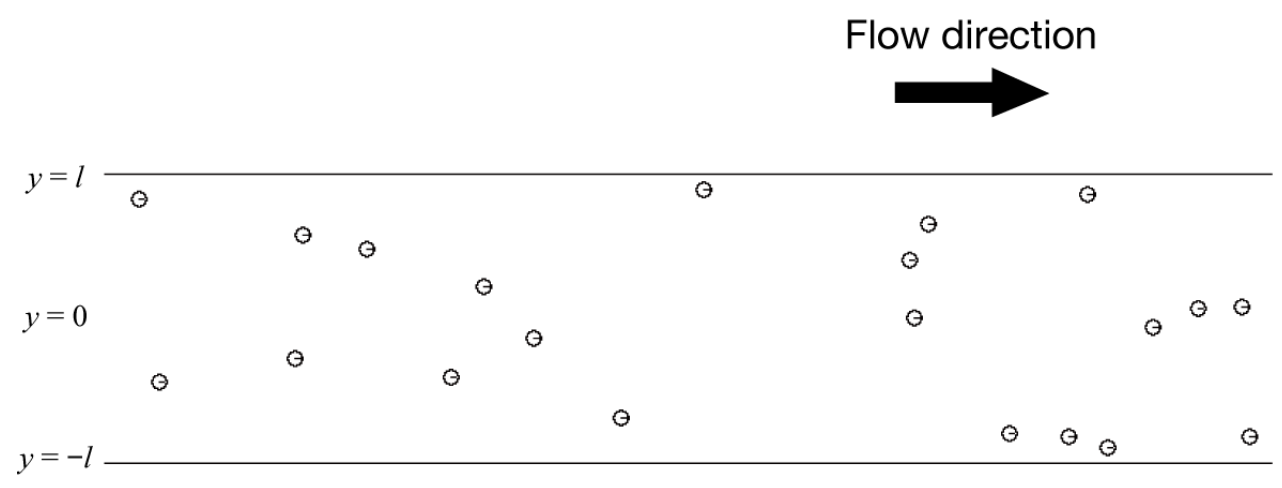

Figure 1: Example of randomly distributed initial condition of suspended particles. Periodic boundary conditions were considered in the axial direction.

Reynolds number $R e$ in eqn (2) was set to 4, 16 and 256 so as to assess inertial effects on the suspended particles and macroscopic non-Newtonian behavior of a suspension

$$
R e=\frac{u_{0} \times 2 l}{v},
$$


where $u_{0}$ is the bulk velocity of the fluid, $2 l$ is the channel width, and $v$ is the kinematic viscosity of the particle-free suspending fluid. The particle Reynolds number $R e_{\mathrm{p}}[6]$ can be defined by taking confinement $C$ into consideration

$$
R e_{\mathrm{p}}=\frac{u_{0} \times 2 r}{v} \times C=\operatorname{Re} \times C^{2},
$$

where $2 r$ is the particle diameter and $C$ is the confinement, which is defined as $C=2 r / 2 l$. Since the confinement $\mathrm{C}$ was set 0.05 in this simulation, the particle Reynolds number $R e_{\mathrm{p}}$ corresponded to $0.01(R e=4), 0.04(R e=16)$, and $0.64(R e=256)$, respectively.

The spatial resolutions $\Delta x$ and $\Delta y$ were set $1 \mu \mathrm{m}(0.3125 \mu \mathrm{m}$ for $R e=256)$, which were validated in our previous researches [7], [8], to obtain high accuracy, and the curved boundaries of the suspended particles were described by virtual flux method [9] in a Cartesian grid. One of the advantages of the virtual flux method is its superior spatial resolution without adding an external force to describe an arbitrary body shape. Additionally, no iterative calculations are necessary in this method. So far, we have successfully applied this method to, for example, internal flow simulation in artery by Navier-Stokes equations [10], and external flow simulation around Savonius rotor by regularized lattice Boltzmann equation [11].

The suspension flow simulations were conducted to the nondimensional time $T$ of 100 , which corresponded to particles travelling distance of $40 \mathrm{~mm}$ on average (100 times as long as the channel width $2 l$ ), so as to assess the temporal change in its microstructure. Since initial particles position more or less affected the temporal changes of the microstructure, these simulations were repeatedly carried out with different randomly dispersed initial positions 30 times or more to reduce their initial dependences. The microstructure of a suspension was then evaluated by its concentration profiles, which can be obtained by counting number of particles scattered in the width direction of the channel.

\subsection{Governing equation for fluid}

Regularized lattice Boltzmann method [12], [13] was used as a governing equation for the fluid part. In this method, the moments were used up to the second order to obtain adequate accuracy. The distribution function $f_{\alpha}$ in the regularized lattice Boltzmann is therefore written as

$$
f_{\alpha}=\omega_{\alpha}\left(a_{0}+b_{i} e_{\alpha i}+c_{i j} e_{\alpha i} e_{\alpha j}\right),
$$

where $\omega$ is the weight factor, $\alpha$ is the direction of the discrete velocity vector $\boldsymbol{e}_{\alpha}$ as shown in Fig. 2, and $a_{0}, b_{i}$, and $c_{i j}$ are the parameters which satisfy the following relationships

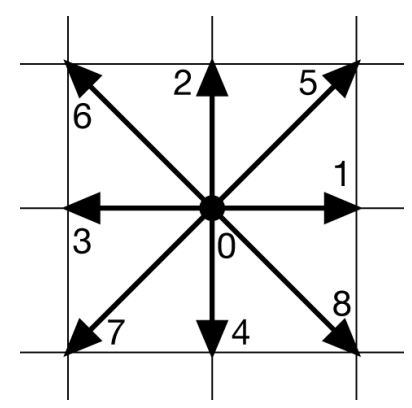

Figure 2: Direction of the discrete velocity vector $\boldsymbol{e}_{\alpha}$ 


$$
\begin{gathered}
\sum_{\alpha} f_{\alpha}=\rho, \\
\sum_{\alpha} e_{\alpha i} f_{\alpha}=\rho u_{i}, \\
\sum_{\alpha} e_{\alpha i} e_{\alpha j} f_{\alpha}=\frac{c^{2}}{3} \rho \delta_{i j}+\rho u_{i} u_{j}+\Pi_{i j}^{\mathrm{neq}},
\end{gathered}
$$

where $\Pi_{i j}^{\text {neq }}$ is the nonequilibrium part of the stress tensor. The distribution function $f_{\alpha}$ is therefore

$$
f_{\alpha}=\omega_{\alpha} \rho\left[1+\frac{3\left(e_{\alpha i} u_{i}\right)}{c^{2}}+\frac{9\left(e_{\alpha i} u_{i}\right)^{2}}{2 c^{4}}-\frac{3\left(u_{i} u_{i}\right)}{2 c^{2}}\right]+\frac{9 \omega_{\alpha}}{2 c^{2}}\left(\frac{e_{\alpha i} e_{\alpha j}}{c^{2}}-\frac{1}{3} \delta_{i j}\right) \Pi_{i j}^{\mathrm{neq}} .
$$

The first term of the right-hand side is equal to Maxwell equilibrium distribution function $f_{\alpha}$ eq, which is a good approximation for low Mach number conditions. The second term of the right-hand side is then substituted for $f_{\alpha}{ }^{1}$, the distribution function $f_{\alpha}$ is written as

$$
f_{\alpha}=f_{\alpha}^{\mathrm{eq}}+f_{\alpha}^{1} .
$$

Finally, the time evolution equation for the regularized lattice Boltzmann equation is then

$$
f_{\alpha}\left(t+\Delta t, \boldsymbol{x}+\boldsymbol{e}_{\alpha} \Delta t\right)=f_{\alpha}^{\mathrm{eq}}(t, \boldsymbol{x})+\left(1-\frac{1}{\tau}\right) f_{\alpha}^{1}(t, \boldsymbol{x})
$$

where $\tau$ is the relaxation time. It has been shown that the Navier-Stokes equations can be derived from the lattice Boltzmann equation through Chapman-Enskog expansion procedure in the incompressible limit [14] with a relaxation time $\tau$ as

$$
\tau=\frac{3 v}{c \delta x}+\frac{\delta t}{2}
$$

where $v$ is the kinematic viscosity. The relaxation time $\tau$ was set 0.74 for all our computations. The distribution function of the D2Q9 model [15] has three different speeds according to its direction as shown in Fig. 2

$$
\begin{array}{cc}
\left|\boldsymbol{e}_{\alpha}\right|=0, & (\alpha=0), \\
\left|\boldsymbol{e}_{\alpha}\right|=c, & (\alpha=1,2,3,4), \\
\left|\boldsymbol{e}_{\alpha}\right|=\sqrt{2} c, \quad(\alpha=5,6,7,8),
\end{array}
$$

where $c=\delta x / \delta t$. The weight factors $\omega_{\alpha}$ for the D2Q9 model are

$$
\begin{aligned}
& \omega_{\alpha}=\frac{4}{9}, \quad(\alpha=0), \\
& \omega_{\alpha}=\frac{1}{9}, \quad(\alpha=1,2,3,4), \\
& \omega_{\alpha}=\frac{1}{36} . \quad(\alpha=5,6,7,8) .
\end{aligned}
$$




\subsection{Governing equations for suspended particles}

Since suspended particles were assumed to be rigid and spherical, their movements were simply described by Newton's second law of motion and the equation of angular motion

$$
\begin{aligned}
& \boldsymbol{F}_{\mathrm{p}}=\rho \frac{d^{2} \boldsymbol{x}_{\mathrm{p}}}{d t^{2}}, \\
& T_{\mathrm{p}}=I \frac{d^{2} \theta_{\mathrm{p}}}{d t^{2}},
\end{aligned}
$$

where $\boldsymbol{F}_{\mathrm{p}}$ is the external hydrodynamic force vector acting on the particle, $\rho$ is the particle density, $\boldsymbol{x}_{\mathrm{p}}$ is the particle position vector, $T_{\mathrm{p}}$ is the torque, $I$ is the moment of inertia, and $\theta_{\mathrm{p}}$ is the particle angle. Note that the density of the suspended particles was equivalent to that of the suspending fluid for neutral buoyancy. The external force vector $\boldsymbol{F}_{\mathrm{p}}$ and the torque $T_{\mathrm{p}}$ acting on the particles were then discretized by a third-order Adams-Bashforth method and solved numerically by a two-way coupling scheme [5]

$$
\begin{gathered}
\dot{\boldsymbol{x}}_{\mathrm{p}}^{n+1}=\dot{\boldsymbol{x}}_{\mathrm{p}}^{n}+\Delta t \frac{23 \boldsymbol{F}_{\mathrm{p}}^{n}-16 \boldsymbol{F}_{\mathrm{p}}^{n-1}+5 \boldsymbol{F}_{\mathrm{p}}^{n-2}}{12 \rho}, \\
\boldsymbol{x}_{\mathrm{p}}^{n+1}=\boldsymbol{x}_{\mathrm{p}}^{n}+\Delta t \frac{5 \dot{\boldsymbol{x}}_{\mathrm{p}}^{n+1}+8 \dot{\boldsymbol{x}}_{\mathrm{p}}^{n}-\dot{\boldsymbol{x}}_{\mathrm{p}}^{n-1}}{12} \\
\dot{\theta}_{\mathrm{p}}^{n+1}=\dot{\theta}_{\mathrm{p}}^{n}+\Delta t \frac{23 T_{\mathrm{p}}^{n}-16 T_{\mathrm{p}}^{n-1}+5 T_{\mathrm{p}}^{n-2}}{12 I} \\
\theta_{\mathrm{p}}^{n+1}=\theta_{\mathrm{p}}^{n}+\Delta t \frac{5 \dot{\theta}_{\mathrm{p}}^{n+1}+8 \dot{\theta}_{\mathrm{p}}^{n}-\dot{\theta}_{\mathrm{p}}^{n-1}}{12} .
\end{gathered}
$$

\section{RESULTS AND DISCUSSION}

Snapshots of pressure-driven suspension flows at nondimensional time $T=100$ are shown in Fig. 3. The suspended particles flowed without collisions or contacts during the simulations. As shown in Fig. 3, flow patterns in the width direction were dependent on the Reynolds number, i.e., inertial effects on the suspended particles. For the case Reynolds number $R e=4$, particles flowed with spreading uniformly in the width direction. In our previous experimental study [16], [17], we have shown these uniform concentration profiles under lower Reynolds number conditions, in which lift force of the particles is negligible. For the case $R e=16$, on the other hand, particles flowed with migrating widthwise toward specific regions around $y= \pm 0.5 \mathrm{l}$. When Reynolds number increased, inertial force acting on the particles increased and widthwise equilibrium positions, where lift force on the particles and repulsive force from the channel wall were equivalent, obviously emerged. Owing to these effects, particles flowed with migrating as Reynolds number increased. This is known as Segré-Silberberg effect [18]. For the case $R e=256$, particles flowed on the centerline of the channel. This is because repulsive forces from the channel wall increased more remarkably than lift forces on the particles. Therefore, the equilibrium position of the particles shifted toward center of the channel. This is known as axial accumulation by sigma effect [19]. 


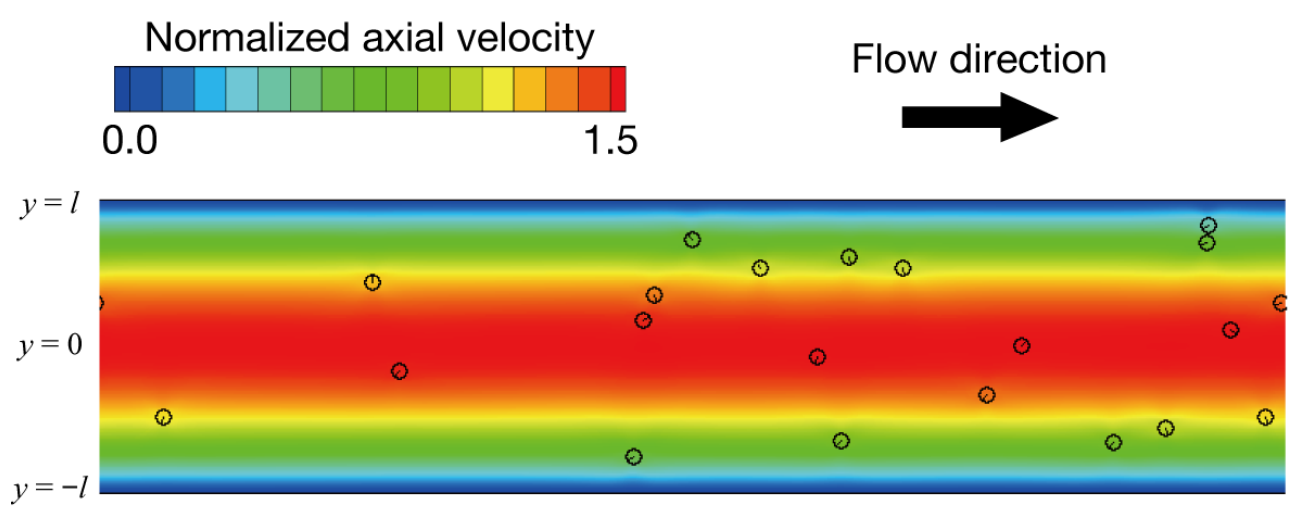

(a) $R e=4\left(R e_{\mathrm{p}}=0.01\right)$

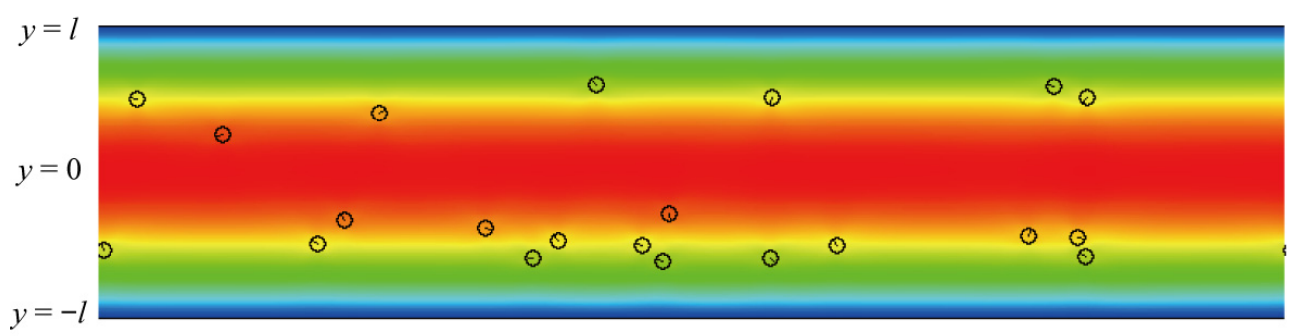

(b) $R e=16\left(R e_{\mathrm{p}}=0.04\right)$

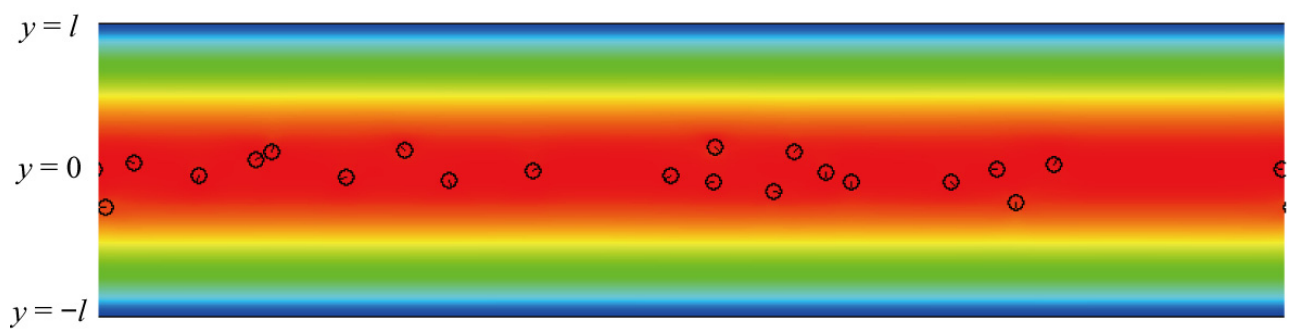

(c) $R e=256\left(R e_{\mathrm{p}}=0.64\right)$

Figure 3: Snapshots of pressure-driven suspension flows at nondimensional time $\mathrm{T}=100$. The flow direction is from left to right. (a) Reynolds number $\mathrm{Re}=4$; (b) Reynolds number $R e=16$; and (c) Reynolds number $R e=256$, respectively.

The concentration profiles are presented in Figs 4-6 as functions of probability density function $\sigma(\mathrm{PDF})$ in order to consider the microstructure of suspensions. The data are the mean \pm 1 standard error (SE). When suspended particles are homogeneously dispersed in the width direction, PDF $\sigma$ exhibits 0.05 as shown by thick black lines in Figs 4-6. For the case $R e=4$, the concentration profiles almost followed the thick black lines and maintained its microstructure in time, which indicates particles flowed homogeneously in the width direction. In this case, the microstructure of suspension was assessed as uniform and steady. 


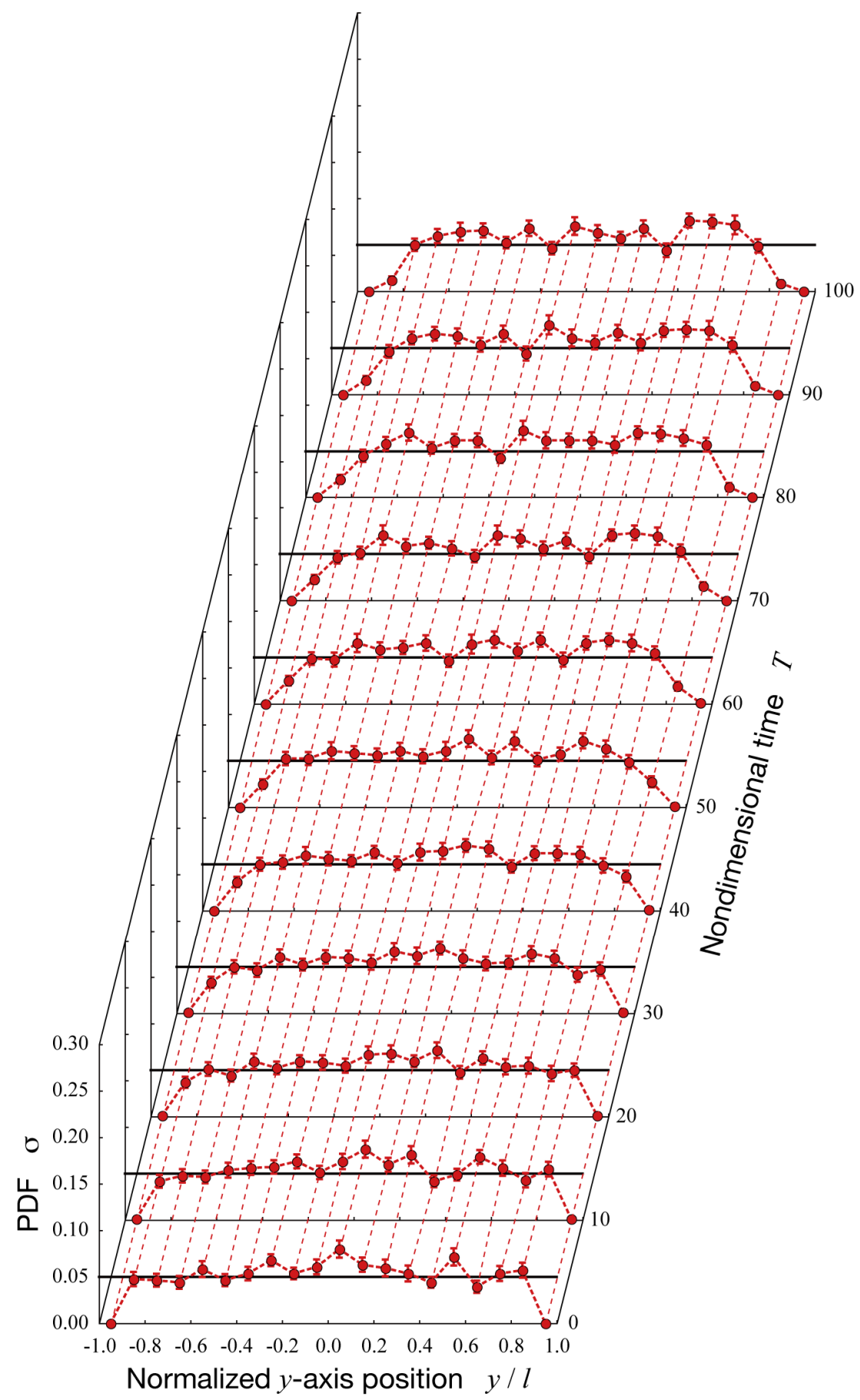

Figure 4: Temporal changes in concentration profile for $R e=4$. The data are the mean \pm 1 SE. The thick black lines (PDF $\sigma=0.05$ ) correspond to homogeneously dispersed states. 


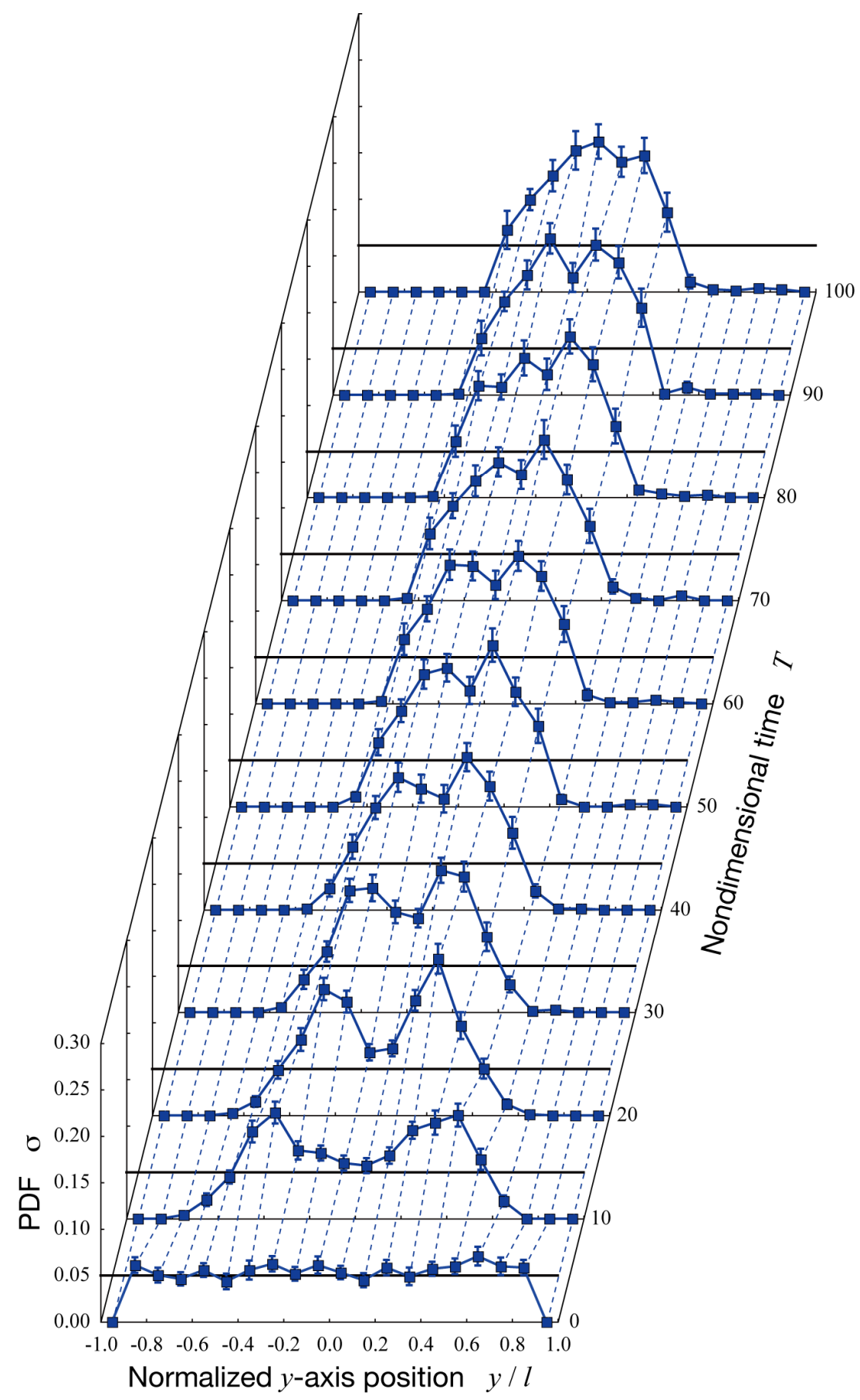

Figure 5: Temporal changes in concentration profile for $R e=256$. The data are the mean \pm 1 SE. The thick black lines (PDF $\sigma=0.05$ ) correspond to homogeneously dispersed states. 


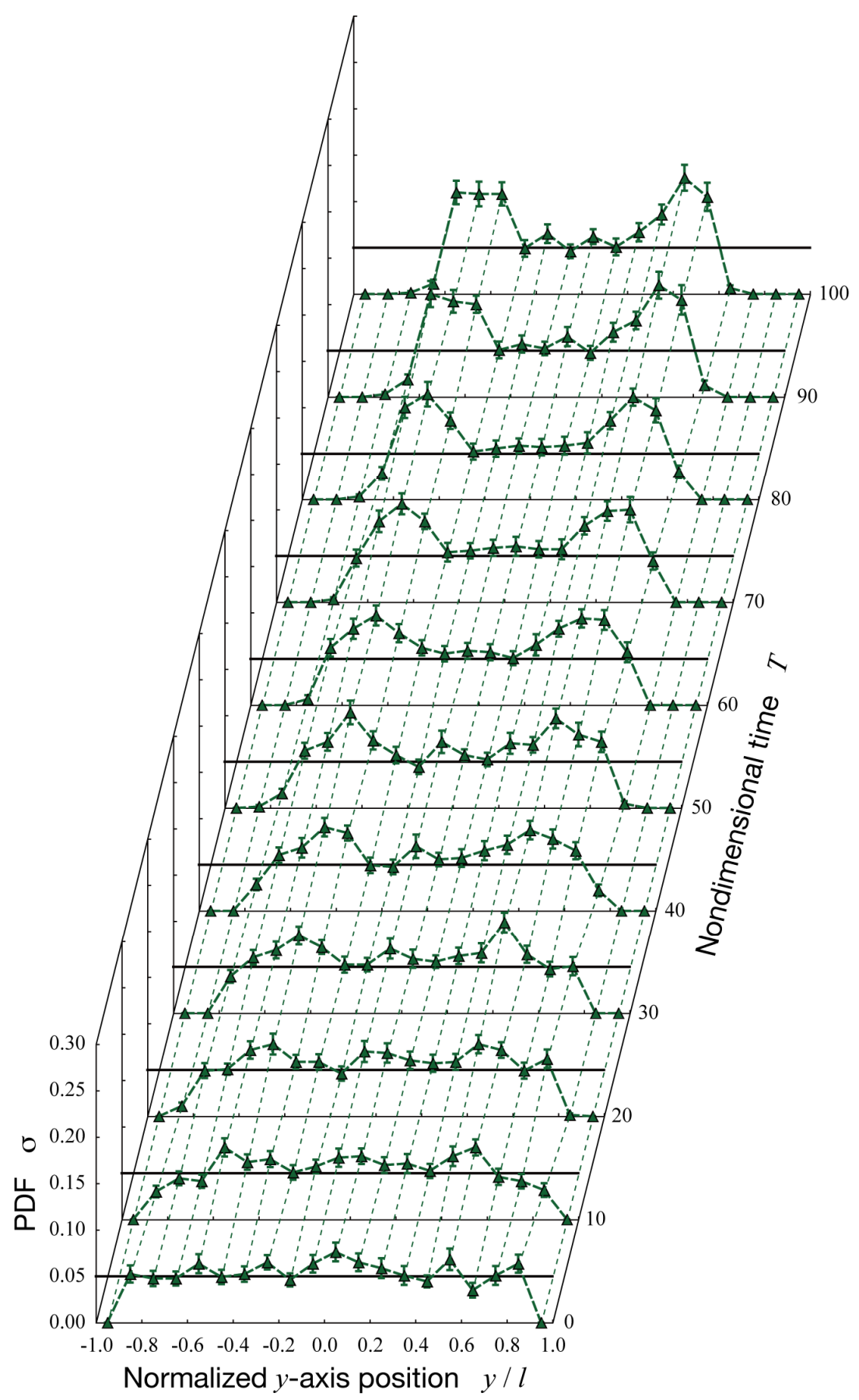

Figure 6: Temporal changes in concentration profile for $R e=16$. The data are the mean \pm 1 SE. The thick black lines (PDF $\sigma=0.05$ ) correspond to homogeneously dispersed states. 
For the case $R e=16$, the concentration profiles gradually left the thick black lines in time, and exhibited two distinct peaks around $y= \pm 0.5 \mathrm{l}$ as shown in Fig. 5. This is because suspended particles gradually migrated toward the widthwise equilibrium positions in time, and the particle concentration became locally high there. Note that the PDF $\sigma$ around the centerline remained flat and unchanged in time, whereas those near the channel walls decreased to zero. Therefore, the peaks of the PDF $\sigma$ around $y= \pm 0.5 l$ were nearly doubled owing to those particles near the walls. This equilibrium migrating position was firstly proposed by Segré and Silberberg [18]. In this case, the microstructure of suspension was considered to be unsteady.

For the case $R e=256$, the concentration profiles changed significantly in time as shown in Fig. 6. First, they showed two peaks around nondimensional time $T=10$ as those for $R e=16$ exhibited. These two peaks then approached each other and made axial accumulation on the centerline. This phenomenon is usually observed in microcirculation known as Fahraeus-Lindqvist effect [19], in which red blood cells flow on the centerline and sufficient plasma layer emerges near the tube wall. Suspended particles and suspending fluid are clearly divided into two layers, which consequently decreases the apparent effective viscosity.

To compare the concentration profile, relationship between PDF $\sigma$ and normalized $y$-axis position $y / l$ at the nondimensional time $T=100$ is shown in Fig. 7. The data are the mean \pm 1 $\mathrm{SE}$. The concentration profile clearly depended on the Reynolds number $R e$. This is because the equilibrium widthwise position depends on inertial force. Peak of the PDF became higher for higher Reynolds number, i.e., nearly doubled for $R e=16$ and tripled for $R e=256$ compared to that of the homogeneous dispersed states.

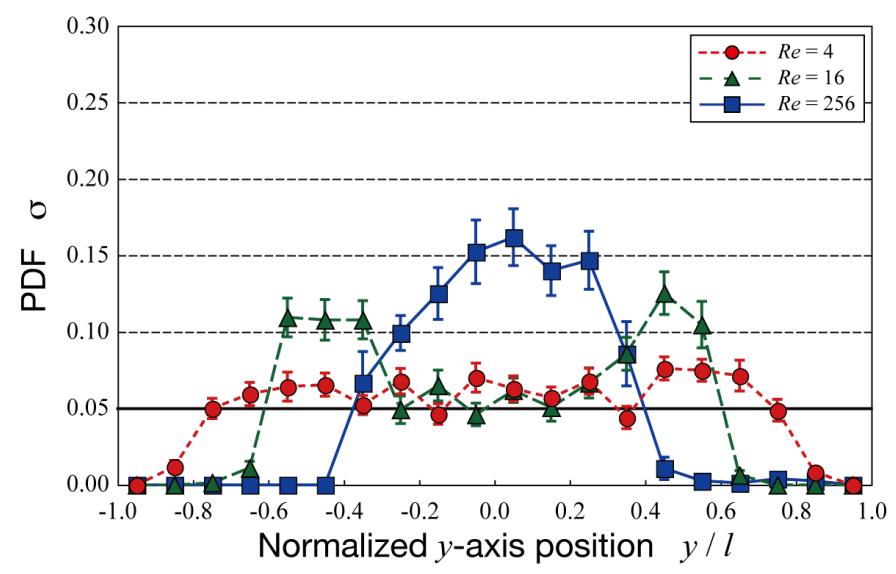

Figure 7: Concentration profiles at the nondimensional time $T=100$. The data are the mean \pm 1 SE. The thick black lines (PDF $\sigma=0.05$ ) correspond to homogeneously dispersed states.

To assess the apparent effective viscosity changes, i.e., thixotropic behavior of suspension, owing to these microstructure changes, relationship between relative viscosity $\eta_{\text {eff }} / \eta_{0}$ and Reynolds number $R e$ is illustrated in Fig. 8. The data are the mean \pm 1 standard deviation (SD). The thick black line in Fig. 8 denotes estimation by Einstein's equation. The relative viscosity for $R e=4$ was compatible with that of Einstein's estimation, in which suspended particles were almost dispersed homogeneously. On the other hand, the 
relative viscosity decreased as Reynolds number increased, in which microstructures of suspension were no longer homogeneous due to inertial effects. Especially, for the case $R e=256$, the relative viscosity almost decreased to 1.0 , i.e., nearly equivalent to that of the suspending fluid. The decrease in relative viscosity was mainly attributed to less particles near the channel wall. Fahraeus-Lindqvist effect was also observed under higher Reynolds number conditions.

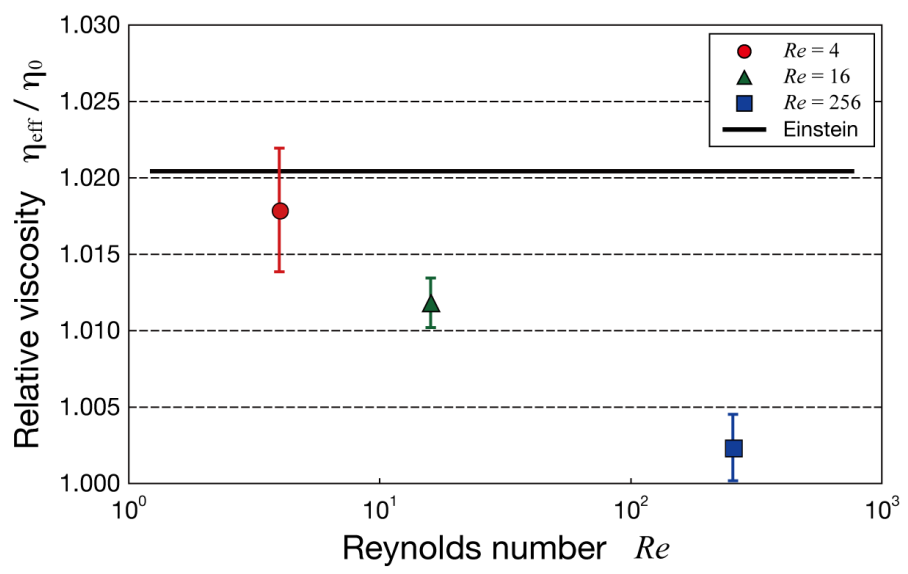

Figure 8: Relationship between relative viscosity $\eta_{\text {eff }} / \eta_{0}$ and Reynolds number $R e$. The data exhibit the mean $\pm 1 \mathrm{SD}$. The thick black line denotes estimation by Einstein's viscosity equation.

\section{CONCLUSIONS}

Pressure-driven suspension flow simulations were carried out to consider the effects of microstructure on the rheological properties of a suspension. As a result, microstructure of a suspension was observed to change in time mainly due to inertial effects of the suspended particles. The thixotropic behavior of a suspension due to inertial effects was successfully reproduced by considering changes in its microstructure. These findings are essential to consider and control rheological properties of a suspension.

\section{ACKNOWLEDGEMENT}

This work was supported in part by JSPS KAKENHI Grant Number JP20K04266.

\section{REFERENCES}

[1] Stickel, J.J. \& Powell, R.L., Fluid mechanics and rheology of dense suspensions. Annual Review of Fluid Mechanics, 37, pp. 129-149, 2005.

[2] Einstein, A., Eine neue bestimmung der molekuldimensionen. Annals of Physics, 19, pp. 289-306, 1906.

[3] Brady, J.F., The Einstein viscosity correction in n dimensions. International Journal of Multiphase Flow, 10, pp. 113-114, 1984.

[4] Doyeux, V., Priem, S., Jibuti, L. \& Farutin, A., Effective viscosity of two-dimensional suspensions: Confinement effects. Physical Review Fluids, 1, 043301, pp. 1-22, 2016. 
[5] Fukui, T., Kawaguchi, M. \& Morinishi, K., A two-way coupling scheme to model the effects of particle rotation on the rheological properties of a semidilute suspension. Computers \& Fluids, 173, pp. 6-16, 2018.

[6] Miura, K., Itano, T. \& Sugihara-Seki, M., Inertial migration of neutrally buoyant spheres in a pressure-driven flow through square channels. Journal of Fluid Mechanics, 749, pp. 320-330, 2014.

[7] Fukui, T., Kawaguchi, M. \& Morinishi, K., Numerical study on the inertial effects of particles on the rheology of a suspension. Advances in Mechanical Engineering, 11, pp. 1-10, 2019.

[8] Fukui, T., Kawaguchi, M. \& Morinishi, K., Relationship between macroscopic rheological properties and microstructure of a dilute suspension by a two-way coupling numerical scheme. Proceedings of the ASME-JSME-KSME 2019 Joint Fluids Engineering Conference, 5449, pp. 1-6, 2019.

[9] Morinishi, K. \& Fukui, T., An Eulerian approach for fluid-structure interaction problems. Computers \& Fluids, 65, pp. 92-98, 2012.

[10] Fukui, T., Asama, H., Kimura, M., Itoi, T. \& Morinishi, K., Influence of geometric changes in the thoracic aorta due to arterial switch operations on the wall shear stress distribution. The Open Biomedical Engineering Journal, 11, pp. 9-16, 2017.

[11] Matsui, T., Fukui, T. \& Morinishi, K., Computational fluid dynamics on a newly developed Savonius rotor by adding sub-buckets for increase of the tip speed ratio to generate higher output power coefficient. Journal of Fluid Science and Technology, 15(2), 0009, pp. 1-16, 2020.

[12] Izham, M., Fukui, T. \& Morinishi, K., Application of regularized lattice Boltzmann method for incompressible flow simulation at high Reynolds number and flow with curved boundary. Journal of Fluid Science and Technology, 6, pp. 812-821, 2011.

[13] Morinishi, K. \& Fukui, T., Parallel computation of turbulent flows using moment base lattice Boltzmann method. International Journal of Computational Fluid Dynamics, 30, pp. 363-369, 2016.

[14] Sterling, J.D. \& Chen, S., Stability analysis of lattice Boltzmann methods. Journal of Computational Physics, 123, pp. 196-206, 1996.

[15] Qian, Y.H., d'Humieres, D. \& Lallemand, P., Lattice BGK models for Navier-Stokes equation. Europhysics Letters, 17, pp. 479-84, 1992.

[16] Kawaguchi, M., Fukui, T., Funamoto, K., Miyauchi, S. \& Hayase, T., Experimental study on the effects of radial dispersion of spherical particles on the suspension rheology. Proceedings of the ASME-JSME-KSME 2019 Joint Fluids Engineering Conference, 5322, pp. 1-6, 2019.

[17] Kawaguchi, M. et al., Viscosity estimation of a suspension with rigid spheres in circular microchannels using particle tracking velocimetry. Micromachines, 10(10), 675, pp. 1-13, 2019.

[18] Segre, G. \& Silberberg, A., Behaviour of macroscopic rigid spheres in Poiseuille flow, Part 2. Experimental results and interpretation. Journal of Fluid Mechanics, 14, pp. 136-157, 1962.

[19] Fahraeus, R. \& Lindqvist, T., The viscosity of the blood in narrow capillary tubes. American Journal of Physiology, 96(3), pp. 562-568, 1931. 